\title{
Multi-sensor Synergy Life Detection Technique Based on Extended D-S Rule
}

\author{
Jingsong Yang ${ }^{a}$, Xiuling $\mathrm{He}^{\mathrm{b}}$ \\ Department of Disaster Prevention Instrument, Institute of Disaster Prevention,Sanhe, 605201, \\ China \\ aemail:yangjingsong@cidp.edu.cn, bemail: hexiuling@cidp.edu.cn
}

Keywords: life detection; multi-sensor synergy; information fusion algorithm; extended D-S rule

\begin{abstract}
A kind of information fusion algorithm is designed based on extended D-S rule for multi-sensor synergy detection, in order to solve the multi-source information fusion problem of uncertainty and conflict in life recognition. First, multi-sensor synergy life detection platform structure is given. Second, the D-S rule and extended D-S rule are used to solve decision level data fusion. Finally, we complete the information fusion recognition of infrared information and acoustic. By comparative analysis, the effectiveness of extended D-S rule is verified in the life detection.
\end{abstract}

\section{Introduction}

The multi-sensor synergy life detection technology is a life information fusion which obtained from multi-source. This technology can produce more effective and more accurate estimates of the living organisms than any single source of information [1]. Similar or different kinds of sensors are used to provide comprehensive information, which can make up for the limitation of single sensor [2][3]. The D-S theory is suitable for information fusion of without a priori, and it has advantages on the uncertainty representation, measurement and combination, at the same time it accords with human reasoning decision-making process. But in the case of high conflict evidence, evidence theory can produce rather counter-intuitive conclusion. A lot of literatures have indicatedd that it is caused by composition rules, and improved composition rules, but the effect is not ideal [4-10].

In this paper, we extend the D - S fusion rules, and regard the conflict as a form of information. Research shows that extension rules can not only make high conflict evidence for reasonable fusion results, but also it can merge the conflict information even which the general basic probability is 1 . The algorithm has strong robustness, and has no additional conditions compared with D - S rules. The simulation results show that the extended D-S rule not only can expansion the use range, but also the algorithm is effective.

\section{D-S combination rules and the extended D-S rules}

Combination rules reflect the combination of a law of evidence. In the following, we assume that $\Theta=\left\{\theta_{1}, \ldots, \theta_{\mathrm{n}}\right\}$ is a finite set (called frame) of $\mathrm{n}$ exhaustive elements. If $\Theta=\left\{\theta_{1}, \ldots, \theta_{\mathrm{n}}\right\}$ is a priori not closed ( $\Theta$ is said to be an open world/frame). $B_{\mathrm{el} 1}$ and $B_{\mathrm{el} 2}$ are reliability functions of the same recognition framework on $\Theta, m_{1}$ and $m_{2}$ are respectively corresponding to the basic reliability distribution. If $A \subseteq \theta$ and $m(A)>0$, then $A$ is called focal element. The focal elements are expressed as $A_{1}, \ldots, A_{\mathrm{k}}$ and $B_{1}, \ldots, B_{l}$. If $\sum_{A_{i} \cap B_{j}=\Phi} m_{1}\left(A_{i}\right) m_{2}\left(B_{j}\right)<1$, then :

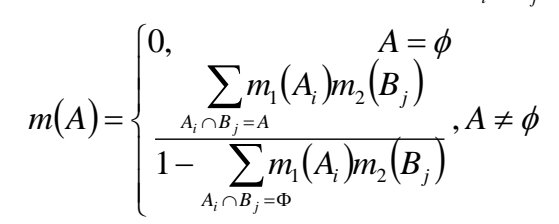

For multiple reliability function, we assume that $B_{\text {el1 }}, \ldots, B_{\text {eln }}$ are reliability functions of the same recognition framework on $\Theta, m_{1}$ and $m_{2}$ are respectively corresponding to the basic reliability distribution. if $\quad B_{\mathrm{el} 1} \oplus, \ldots, \oplus B_{\mathrm{eln}}$ is existence, then for $\forall A \subset \Theta, \quad A \neq \phi$ : 


$$
m(A)=k \sum_{\substack{A_{1}, \ldots A_{n} \in \Theta \\ A_{1} \cap, A_{2} \ldots \cap A_{n}=A}} m_{1}\left(A_{1}\right), \ldots m_{n}\left(A_{n}\right),
$$

Where :

$$
k=\left[\sum_{\substack{A_{1}, \ldots A_{n} \in \Theta \\ A_{1}, A_{2}, \ldots \cap A_{n} \neq A}} m_{1}\left(A_{1}\right), \ldots m_{n}\left(A_{n}\right)\right]^{-1}
$$

It can be seen that the combination of multiple evidence has nothing to do with the order from equation (1) and (2). So the multiple evidence combination can be obtained with two evidence combination of recursive calculation.

In general, D-S rules are more suitable to solve the fusion with high confidence and low confliction, but for high conflict it can produce all kinds of unreasonable results. Because D-S rules structure the confidence interval based on the information which have no conflict, it abandoned every conflict of independent focal element. It is easy to see that ignore the conflict for normalization is a kind of adventure. It will produce some contrary example to intuition. Especially when $\mathrm{K}=1$, D-S rule cannot be used, so robustness of D-S rule is not strong. How to improve the defects of D - S rules has extremely important significance in practical application.

Conflicts are also a kind of information. The principle of extended D-S rules is to let conflict information join fusion rules after extraction and analysis. Specific operation rules are as follows: when the basic probability assignment is conflicting, $\mathrm{K}$ ( $\mathrm{K}$ is said the probability of total conflict) is distributed to the conflict focal element. The number of $\mathrm{K}$ is according to the percentage of the conflict focal element in the total basic probability assignment conflict. We put more the conflict probability assignment to the focal element which own larger probability assignment, because the probability assignment of Interrupted focal element is smaller than undisturbed. Therefore, the fusion rules can be transformed into:

$$
m(A)=\left\{\begin{array}{c}
0, \quad A=\phi \\
\sum_{A_{A} \cap B_{j}=A} m_{1}\left(A_{i}\right) m_{2}\left(B_{j}\right)+f(A)
\end{array}, A \neq \phi\right.
$$

Where $f(A)$ is the contradiction probability distribution function, it satisfy: $f(A) \geqslant 0, f(\phi)=0$, $\sum_{A \subset \Theta} f(A)=K \quad, f(A)=K \cdot q(A)$, where:

$$
\begin{aligned}
& K=\sum_{A_{i} \cap B_{j}=\Phi} m_{1}\left(A_{i}\right) m_{2}\left(B_{j}\right) \\
& q(A)=\frac{\sum_{1 \leq i \leq n} m_{i}(A)}{\sum_{A_{i} \cap B_{i}=C}\left(\sum_{1 \leq i \leq n} m_{i}(C)\right)}, \quad A \subset C, C \neq \Theta, C \neq \phi
\end{aligned}
$$

\section{Multi-sensor life detection platform design}

The synergy detection platform is composed of hardware platform and software platform. The hardware platform includes infrared sensor, vibration sensor, ultrasonic sensor, multi-channel acoustic/vibration data acquisition card, ultrasonic data collection card, embedded processors and I/O devices, as shown in Figure 1. The software platform use the LabView as a development tool, to complete the video and sound information collection, information display and processing.

The infrared image and acoustic information is completely different qualitative information, that is, different information. Usually, different information has the very strong complementary to each other, so the fusion of information produced by the more practical value. However, alien sensor information fusion are faced with many difficulties, the main difficulty is currently there is no general mathematical tools and methods, description and analysis of the different information features and many sources of information fusion corresponding. 


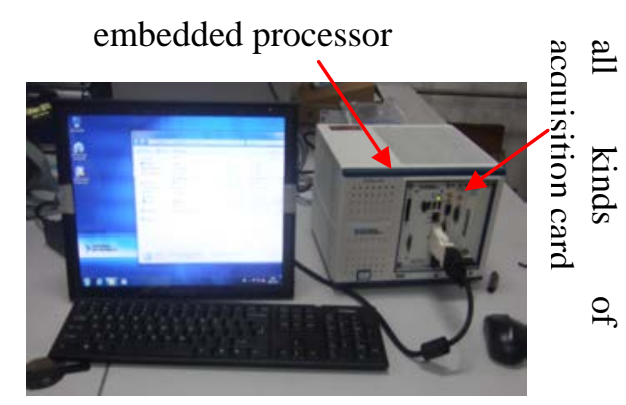

Fig.1. Hardware platform structure

The multiple source information fusion general can carry out in different levels, such as data level, characteristic level and decision level. Because different inherent characteristics of information and is currently in the data layer of fusion extremely hard, general only limited characteristic layer or decision-making for research. And decision-making level fusion is in the top of the said information on the fusion processing.

\section{Application of extended D-S rules in the life detection}

The process of extended D-S rules used to the life detection is : the first, to define the recognition framework; the second computing generalized reliability distribution function; and then to combination of generalized reliability distribution function according to the extended D-S rules; finally, to Identify life according to rules of actual needs.

The processes of using extended D-S rules in life detection are as follows:

Step 1: Define recognition framework, the proposition could be to join recognition framework. We design life recognition framework $\Theta=\{A, B, C\}$, here A for survivors, $B$ is said no survivors, $C$ is said not sure.

Step 2: Get generalized confidence functions, in life recognition; it is given by experts according to the sensors.

Step 3: Use suitable fusion rules to combine the probabilities of generalized confidence functions. The probabilities are calculated by the equation (4) - (6).

Step 4: Determine the life identification decision rules. The synthesis of the decision rules is the maximum reliability, in this paper.

\section{Test results}

In this case, the space of discernment $\Theta$ represents the survivor identification on infrared images and acoustic information. The experts give their perception and belief according to their certainty. Consequently we have to take into account all these aspects of the applications. In order to simplify, we consider only three classes in the following: survivor as A, no-survivors, referred as B, and indetermination, referred as $\mathrm{C}$. Hence, on certain tiles, $\mathrm{A}, \mathrm{B}$, and $\mathrm{C}$ can be present for one or more experts. The belief functions have to take into account the certainty given by the experts (numbers in $[0,1])$ as well as the proportion of the kind of survivor identification.

We give the obtained results on a multi-sensor life detection system for the fusion of two experts in infrared images and acoustic information. Table 1 shows the generalized confidence given by two experts, which $m_{11}$ : confidence given by expert1 based on infrared image; $m_{12}$ : confidence given by expert1 based on acoustic information; $m_{21}$ : confidence given by expert 2 based on infrared image; $m_{22}$ : confidence given by expert2 based on acoustic information.

Table 1. Generalized confidence functions.

\begin{tabular}{|c|c|c|c|c|}
\hline Target type & $\boldsymbol{m}_{\mathbf{1 1}}$ & $\boldsymbol{m}_{\mathbf{1 2}}$ & $\boldsymbol{m}_{\mathbf{2 1}}$ & $\boldsymbol{m}_{\mathbf{2 2}}$ \\
\hline A & 0.5 & 0 & 0.55 & 0.55 \\
\hline B & 0.2 & 0.9 & 0.1 & 0.1 \\
\hline C & 0.3 & 0.1 & 0.35 & 0.35 \\
\hline
\end{tabular}

Calculate order is as follows: first, image data fusion and acoustic information fusion, then theresult of image and sound information fusion for heterogeneous information fusion.

We present here the results reached by the fusion with the combination rule, the D-S rule and the 
extended D-S rule, shown as Table 2.

Table 2. Results with different combination rule.

\begin{tabular}{|c|c|c|}
\hline Results & D-S & extended D-S \\
\hline$m(A)$ & 0 & 0.445331 \\
\hline$m(B)$ & 0.328767 & 0.290854 \\
\hline$m(C)$ & 0.671233 & 0.263815 \\
\hline
\end{tabular}

The results in Table 2 show that D-S rule's result is contrary to intuition, which determine the target identity as "B". Because evidence 3 has "veto", no amount of evidence to support "A", it is a serious error. However, the extended D-S rule determines the target identity as "A", which is satisfied the verdict. It means that the extended D-S rule is suit to solve the problems brought by high conflict evidence.

\section{Conclusion}

The extended D-S rule is generalized extended of D-S theory .It can effectively deal with uncertain information and conflict problem. Decision-making level fusion based on the extended D-S rule is more suitable for complex environment of earthquake relief, and can get more comprehensive, more accurate information of survivors. We have shown on real data that there is a difference of decision following the choice of the combination rule. The results show that the extended D-S rule is suit to solve the problems brought by high conflict evidence.

\section{Acknowledgement}

In this paper, the research was sponsored by the Seismic Technology Spark Plan Foundation of China (Grant No.XH12076); Special Fund of Fundamental Scientific Research Business Expense for Higher School of Central Government (Projects for creation teams) ( Grant No. ZY20110104).

\section{References}

[1] Jing-song YANG, YAO Zhen-jing, SONG Yan-xing, HE Xiu-ling. Design life detection synergy platform with infrared and acoustic wave [J]. CHINA MEASUREMENT \& TEST, 20135 (39) 72-75.

[2] Jingsong Yang, Xiu Ling He, Li Xin Li. Multi-Sensor Life Detection Synergy Platform Design[J]. Applied Mechanics and Materials, 2013, 442 (2014): 520-525

[3] Jingsong YANG, Xun CHEN. Decision-Making Level Fusion Based on DSmT for Multi-Sensor Life Detection Platform[J]. Sensors \& Transducers, 2014, 2(164) :31-35.

[4] L. A. ZEDEH. Review of Shafer's. A mathematical theory of evidence[J]. AI Magazine, 1984, 5(3):81-83.

[5] R. YAGER. On the dempster shafer framework and new combination rules[J]. Information Sciences, 1987,41(2): 93-137.

[6] F. SMARANDACHE, J. DEZERT, Advances and Applications of DSmT for Information Fusion. Rehoboth[M], American Research Press, 2004.

[7] Chen L $\mathrm{H}$. An extended rule-based inference for general decision making problem[J].Information Science, 1997, 102(1 / 4):11-137.

[8] Yager R R。On the Dempster-Shafer frame work and new combination rules[J]. Information System,1989,41(2):93-137.

[9] Horiuchi Takahiko. A new theory of evidence for nonexchsive elementary proposition[J]. International Joumal of System Science,1996,27(10):989-994. HIROKIK, MINORUA, YASUOK, ET al.RoboCup: a challenge problem for AI and robotics. HirokiK. RoboCup-97: Robot Soccer World Cup [C]. Berlin: Springer, 1998.38-43.

[10] LI Hongfeil, TIAN Kangshen, JIN Hongbin, FANG Weihua. A Interactive Combination Method of Target Identity Identification [J]. Journal of Projectiles, Rockets. Missiles and Guidance, 2011,31(1): 207-210. 